\title{
A Coherent System Component Importance under Its Signatures Representation
}

\author{
Vanderlei da Costa Bueno \\ Institute of Mathematics and Statistics, São Paulo University, São Paulo, Brazil \\ E-mail: bueno@ime.usp.br \\ Received April 5, 2011; revised April 29, 2011; accepted May 20, 2011
}

\begin{abstract}
In this paper we discuss how to measure the component importance for a system in its signature representation. The definition is given in terms of compensator transform and it can be considered as a new formalization of the ideas presented by Bergman [1] in the context of system signature.
\end{abstract}

Keywords: System Signature, Dynamic System Signature, Coherent Systems, Component Importance, Point Processes Martingales

\section{Introduction}

The signature of a coherent system with independent and identically distributed component lifetimes, as defined by Samaniego [2], is a vector whose $i$-th coordinate is the probability that the $i$-th component failure is fatal for the system. The key feature of system signatures that makes them broadly useful in reliability analysis is the fact that, in the context of independent and identically distributed (i.i.d.) absolutely continuous components lifetimes, they are distribution free measures of system quality, depending solely on the design characteristics of the system and independent of the behavior of the systems components.

A detailed treatment of the theory and applications of system signatures may be found in Samaniego [2]. This reference gives detailed justification for the i.i.d. assumption used in the definition of system signatures. By the way there are some applications in which the i.i.d. assumption is appropriate, ranging from batteries in lighting, to wafers or chips in a digital computer to the subsystem of spark plugs in an automobile engine.

Samaniego [3], Kochar, et al. [4] and Shaked and Suarez-Llorens [5] extended the signature concept for components exchangeable lifetimes, an interesting and practical situation in reliability theory.

There seems to be two mains reasons for given an importance measure of systems components. Firstly, it permits the analyst to determine which component merits the most additional research and development to improve overall system reliability at minimum cost or effort. Secondly, it may suggest the most efficient way to diag- nose system failure by generating a checklist for an operator to follows.

Birnbaum [6] defined the importance of a component in a system (essentially) as follows. Let $S$ and $T$ denote the random lifetimes of the component and the system, respectively. Then the importance of $S$ for $T$ at time $t$ is

$$
I_{t}^{B}(S, T)=P(T>t \mid S>t)-P(T>t \mid S \leq t) .
$$

Note, however, that this definition is meaningful for any two lifetimes (the coherent system framework is not essential).

This measure depends in a given point in time and it is not quite relevant for most design or redesign decisions. Several time independent importance measures have been suggested, and most of then are weighted integrals of $I_{t}^{B}(S, T)$ over $t$ (e.g. Barlow and Proschan [7], Natvig [8], for a survey see Bolland and El-Neweihi [9]).

Bergman [1] pointed out that many importance measures in reliability theory can be obtained through the study of the change of the system expected lifetime due to different variations of component lifetime distributions. Assume that the components are independent, and let $F_{i}$ and $G_{i}$, denote the original and the modified distribution of component $i$ with respect to this "component improvement” is given as

$$
\int_{0}^{\infty}\left(\bar{G}_{i}(t)-\bar{F}_{i}(t)\right) I_{t}^{B}(S, T) \mathrm{d} t,
$$

where $\bar{F}_{i}(t)=1-F_{i}$. For example, in the case of Natvigs importance measure, the improvement is obtained through 
a minimal repair of the component in question. The Barlow and Proschan reliability importance is obtained from an infinitesimal transformation.

Concerning the signature representation of the system survival lifetime

$$
P(T>t)=\sum_{i=1}^{n} \alpha_{i} P\left(T_{(i)}>t\right),
$$

where $\left\{T_{(i)}, 1 \leq i \leq n\right\}$ are the ordered $\left\{T_{i}, 1 \leq i \leq n\right\}$, the system lifetime is a function of the order statistics and the importance of the $i$-th failure for the system reliability follows standard arguments. However in field operations, the system is a function of its components and it is natural to ask about the component reliability importance for system reliability in its signature representation, that is: What is the reliability importance of the component $j$ for the system reliability at the $i$-th failure? The paper motivation is to answer this main question. Recall that, even if the components are independent and identically distributed, the order statistics are not.

To detect the effects of the independent (exchangeable) components distribution lifetimes transformation in the ordered statistics and in the system signature representation itself, we are going to consider compensator transforms.

It is well known that there exists a bijective relation between the space of all distributions functions and the $\mathfrak{I}_{t}$-compensators space characterized by the so called Doléans exponential equation

$$
\bar{F}\left(t \mid \mathfrak{I}_{t}\right)=e^{-A^{c}(t)} \prod_{s \leq t}(1+\Delta A(s))
$$

where $A^{c}(t)$ is the continuous part of $A(t)$ and $\Delta A(t)=A(t)-A^{c}(t)$ is its discrete part. We consider dynamics signatures, as in a recent work by Bueno [10], in a general set up, under a complete information level where the dependence (exchangeability) can be considered. In this work, in Section 2 we summarize the results in dynamics signature from [10]. In Subsection 3.1 of Section 3 we develop the Barlow and Proschan importance reliability under the signature representation and in Subsection 2 we develop the component importance through compensator transform.

\section{Dynamic System Signature}

In the following we are going to use the following notation;

$T$ is the system lifetime.

$T_{j}$ is the lifetime of component $j, 1 \leq j \leq n$.

$T_{(i)}$ is the time of the $i$-th order statistics, $1 \leq i \leq n$.

$T_{(i) j}$ is the time of the $i$-th order statistics caused by component $j, 1 \leq i, j \leq n$.
$F$ is the distribution function of the system lifetime.

$F_{j}$ is the distribution function of the $j$-th component lifetime.

$F_{(i) j}$ is the standardized distribution function of $T_{(i) j}$.

$\bar{F}$ is the survival function of the system lifetime.

$\bar{F}_{j}$ is the survival function of the $j$-th component lifetime.

$\bar{F}_{(i) j}=1-F_{(i) j}$.

$\left(T_{(i)}, X_{i}\right)_{i \geq 1}$ is a marked point process.

$X_{i}$ is the Mark corresponding to $T_{(i)}$.

$\left(\mathfrak{J}_{t}\right)_{t \geq 0}$ is the filtration generated by $\left(T_{(i)}, X_{i}\right)_{i \geq 1}$.

$N_{(i)}(t)=1_{\left\{T_{(i)} \leq t\right\}}$ is the counting process of $T_{(i)}$.

$N_{(i) j}(t)=1_{\left\{T_{(i) j} \leq t\right\}}$ is the counting process of $T_{(i) j}$.

$A_{(i)}(t)$ is the $\mathfrak{I}_{t}$-compensator of $N_{(i)}(t)$.

$A_{(i) j}(t)$ is the $\mathfrak{I}_{t}$-compensator of $N_{(i) j}(t)$.

In our general setup, we consider the vector $\left(T_{1}, \cdots, T_{n}\right)$

of $n$ component lifetimes which are finite and positive random variables defined in a complete probability space $(\Omega, \mathfrak{I}, P)$, with $P\left(T_{i} \neq T_{j}\right)=1$, for all $i \neq j, i, j$ in $E=\{1, \cdots, n\}$, the index set of components. The lifetimes can be dependent but simultaneous failures are ruled out. To simplify the notation, we assume that relations such as $\subset,=, \leq,<, \neq$ between random variables and measurable sets, respectively, always hold with probability one, which means that the term Pa.s., is suppressed.

We consider, as in Bueno [10], the system evolution on time under a complete information level. In this fashion, if the components lifetimes are absolutely continuous, independent and identically distributed, the expected dynamic system signature enjoy the special property that they are independent of both the distribution $F$ and the time $t$. Also the dynamic system signature actualizes itself under the system evolution on time recovering the original coherent system signature in the set $\left\{T_{(n)} \leq t\right\}$ as in Samaniego [2].

We denote by $T_{(1)}<T_{(2)}<\cdots<T_{(n)}$ the ordered lifetimes $T_{1}, \cdots, T_{n}$, as they appear in time and by $X_{i}=\left\{j: T_{(i)}=T_{j}\right\}$ the corresponding marks. As a convention we set $T_{(n+1)}=T_{(n+2)}=\cdots=\infty$ and $X_{n+1}=X_{n+2}=\cdots=e$ where $e$ is a fictitious mark not in $E$. Therefore the sequence $\left(T_{(n)}, X_{n}\right)_{n \geq 1}$ defines a marked point process.

The mathematical formulation of our observations is given by a family of sub $\sigma$-Algebras of $\mathfrak{I}$, denoted by $\left(\mathfrak{I}_{t}\right)_{t \geq 0}$, where

$$
\mathfrak{I}_{t}=\sigma\left\{1_{\{T>s\}}, 1_{\left\{T_{(i)}>s\right\}}, X_{i}=j, 1 \leq i \leq n, j \in E, 0<s \leq t\right\},
$$

satisfies the Dellacherie conditions of right continuity and completeness, and $T$ is the system lifetime 


$$
T=\min _{1 \leq j \leq k} \max _{i \in K_{j}} T_{i},
$$

where $K_{j}, 1 \leq j \leq k$ are minimal cut sets, that is, a minimal set of components whose joint failure causes the system fail.

Intuitively, at each time $\mathrm{t}$ the observer knows if the events $\left\{T_{(i)} \leq t, X_{i}=j\right\}(\{T \leq t\})$ have either occurred or not and if they have, he knows exactly the value $T_{(i)}(T)$ and the mark $X_{i}$. We assumed that $T_{1}, \cdots, T_{n}$ are totally inaccessible $\mathfrak{J}_{t}$-stopping time. In a practical sense we can think of a totally inaccessible $\mathfrak{I}_{t}$-stopping time as an absolutely continuous lifetime. For a mathematical basis in stochastic process martingale applied to reliability theory see the book by Aven and Jensen [11].

The simple marked point process $N_{(i) j}(t)=1_{\left\{T_{(i)} \leq t, X_{i}=j\right\}}$ is an $\mathfrak{I}_{t}$-submartingale and from the Doob-Meyer decomposition we know that there exists a unique $\mathfrak{I}_{t}$ predictable process $\left(A_{(i) j}(t)\right)_{t \geq 0}$, called the $\mathfrak{I}_{t}$-compensator of $N_{(i) j}(t)$, with $A_{(i) j}(0)=0$ and such that $N_{(i) j}(t)-A_{(i) j}(t)$, is an $\mathfrak{I}_{t}$-martingale. $A_{(i) j}(t)$ is absolutely continuous by the totally inaccessibility of $T_{i}$, $1 \leq i \leq n$. We also define the lifetime $T_{(i) j}$ through the process

$$
F_{(i) j}(t)=P\left(T_{(i) j}(t) \mid \mathfrak{J}_{t}\right)=P\left(T_{(i)} \leq t, X_{i}=j \mid \mathfrak{I}_{t}\right),
$$

of the $i$-th failure leads by the $j$-th component failure.

The compensator process is expressed in terms of the conditional probability, given the available information and generalizes the classical notion of hazards.

As $N_{(i) j}(t)$ can only count on the time interval $\left(T_{(i-1)}, T_{(i)}\right]$, the corresponding compensator differential $d A_{(i) j}(t)$ must vanish outside this interval. Let $N_{(i)}(t)=\sum_{j \geq 1} N_{(i) j}(t)$ be the $i$-th failure point process with $\mathfrak{I}_{t}$-compensator process $A_{(i)}(t)=\sum_{j \geq 1} A_{(i) j}(t)$. The $\mathfrak{I}_{t}$-compensator of $N_{j}(t)=1_{\left\{T_{j} \leq t\right\}}$, corresponding to the $j$-th components lifetime, is $A_{j}(t)=\sum_{i \geq 1} A_{(i) j}(t)$.

Conveniently, we define the critical level of the component $j$ for the $i$-th failure, $Y_{(i) j}$, as the first time from which onwards the failure of component $j$ lead to system failure at $\left\{T=T_{(i)}, X_{i}=j\right\}$. We consider the $\mathfrak{I}_{t}$-compensator process $\left(A_{\phi}(t)\right)_{t \geq 0}$ of the point process $N_{\phi}(t)=1_{\{T \leq t\}}$, of the system lifetime $T$, such that $N_{\phi}(t)-A_{\phi}(t)$ is a zero mean $\mathfrak{I}_{t}$-martingale with $P(T \leq t)=E\left[N_{\phi}(t)\right]=E\left[A_{\phi}(t)\right]$. Bueno [10], proves the following results:
Theorem 2.1 Under the above notation, in the set $\{T>t\}$, the $\mathfrak{I}_{t}$-compensator of $N_{\phi}(t)=1_{\{T \leq t\}}$, is

$$
A_{\phi}(t)=\sum_{i=1}^{n} \sum_{j=1}^{n}\left[A_{(i) j}(t)-A_{(i) j}\left(Y_{(i) j}\right)\right]^{+} 1_{\left\{T_{(i-1)} \leq t<T_{(i)}\right)},
$$

where $a^{+}=\max \{a, 0\}$.

Theorem 2.2 Let $T$ be the lifetime of a coherent system of order $n$, with component lifetimes $T_{1}, \cdots, T_{n}$ which are totally inaccessible $T_{1}, \cdots, T_{n} \quad \mathfrak{I}_{t}$-stopping time. Then, under the above notation and at complete information level, we have

$$
P\left(T \leq t \mid \mathfrak{J}_{t}\right)=\sum_{i=1}^{n} \sum_{j=1}^{n} \frac{P\left(T=T_{(i)}, X_{i}=j \mid \mathfrak{I}_{t}\right)}{P\left(T \geq T_{(i)} \mid \mathfrak{I}_{t}\right)} 1_{\left\{T_{(i)} \leq t<T_{(i+1)}\right\}},
$$

with $T_{(n+1)}=\infty$.

Remarks 2.1 1) In the case of independent and identically distributed lifetimes we have

$$
P\left(T \leq t \mid \mathfrak{J}_{t}\right)=\sum_{i=1}^{n} \frac{P\left(T=T_{(i i)}\right)}{P\left(T \geq T_{(i)}\right)} 1_{\left\{T_{(i i)} \leq t<T_{(i+1)}\right\}} .
$$

2) Clearly, it is not seemingly true to think the general case of dependent components in the signatures context. However, as Shaked et al., [5], asked, it is plausible to analyse the case of dependent and identically distributed lifetimes (any way, its holds true for exchangeable distribution). In this case we have

$$
P\left(T \leq t \mid \mathfrak{J}_{t}\right)=\sum_{i=1}^{n} \frac{P\left(T=T_{(i i)} \mid \mathfrak{I}_{t}\right)}{P\left(T \geq T_{(i i)} \mid \mathfrak{J}_{t}\right)} 1_{\left\{T_{(i i)} \leq t<T_{(i)+1}\right\}} .
$$

Clearly, in the case of exchangeability, the expression in 1) is holding.

Corollary 2.1 Let $T$ be the lifetime of a coherent system of order $n$, with component lifetimes $T_{1}, \cdots, T_{n}$ which are independent and identically distributed with continuous distribution $F$. Then,

$$
P\left(T \leq t \mid \mathfrak{J}_{t}\right)=\sum_{i=1}^{n} \beta_{i} 1_{\left\{T_{(i)} \leq t\right\}}
$$

where

$$
\beta_{i}=\frac{P\left(T=T_{(i)}\right)}{P\left(T \geq T_{(i)}\right)}-\frac{P\left(T=T_{(i-1)}\right)}{P\left(T \geq T_{(i-1)}\right)},
$$

with $T_{(0)}=0, T_{(n+1)}=\infty$, and $\sum_{i=1}^{n} \beta_{i}=1$.

Definition 2.1 Let $T$ be the lifetime of a coherent system of order $n$, with component lifetimes $T_{1}, \cdots, T_{n}$ which are independent and identically distributed random variables with absolutely continuous distribution $F$. 
Then the dynamic signature vector $\beta$ is defined as

$$
\beta=\left(\beta_{1}, \cdots, \beta_{n}\right)
$$

if

$$
\beta_{i}=\frac{P\left(T=T_{(i)}\right)}{P\left(T \geq T_{(i)}\right)}-\frac{P\left(T=T_{(i-1)}\right)}{P\left(T \geq T_{(i-1)}\right)} \geq 0,
$$

and the $T_{(i)}$ are the order statistics of $T_{i}, 1 \leq i \leq n$.

\section{Component Importance}

\subsection{The Barlow and Proschan Component Importance}

Considering the classical Barlow and Proschan component importance reliability for system reliability, as in [7], the importance of the component $i$ is the probability that the system failure coincides with the failure of the $i$-th component, that is

$$
\begin{aligned}
I(i) & =P\left(T=T_{i}\right) \\
& =\int_{0}^{\infty}\left[P\left(T>s \mid T_{i}>s\right)-P\left(T>s \mid T_{i} \leq s\right)\right] \mathrm{d} F_{i}(s)
\end{aligned}
$$

in which case, under the iid lifetimes assumption, we have the Barlow and Proschan structural reliability, it should be natural to measure the reliability importance of the $i$-th failure by $I(i)=P\left(T=T_{(i)}\right)=\alpha_{i}$ in a similar fashion.

However we are addressing another question. As before system realization we only know the component lifetimes (we does not know the order statistics), the important question about the component reliability structural importance for system reliability in its signature representation is: What is the reliability importance of the component $j$ for the system reliability at the $i$-th failure? We consider the following definition.

Definition 3.1.1 Let $T$ be the lifetime of a coherent system of components identically distributed and let $T_{(i) j}$ be the $i$-th failure lifetime caused by the component $j$, with $\mathfrak{I}_{t}$-compensator process $\left(A_{(i) j}(t)\right)_{t \geq 0}$. The reliability importance of component $j$ to system reliability at the $i$-th failures is

$$
I_{(i) j}=E\left\{\left[A_{(i) j}(T)-A_{(i) j}\left(Y_{(i) j}\right)\right]^{+}\right\},
$$

Where $Y_{(i) j}$ is the critical level of the component $j$ for the $i$-th failure.

The reliability importance of the $i$-th failure to system reliability is

$$
I(i)=\sum_{j=1}^{n} I_{(i) j}
$$

Proposition 3.1.1 Let $T$ be the lifetime of a coherent system of components independent and identically distributed and let $T_{(i) j}$ be the $i$-th failure lifetime caused by the component $j$, with $\mathfrak{I}_{t}$-compensator process $\left(A_{(i) j}(t)\right)_{t \geq 0}$ where $A_{(i) j}(t)=-\ln \bar{F}_{(i) j}\left(t \wedge T_{(i) j}\right)$ and $F_{(i) j}(t)=1-\bar{F}_{(i) j}(t)$, (see Arjas and Yashin [12]). Then, the reliability importance of component $j$ to the system reliability at the $i$-th failures is

$$
I_{(i) j}=P\left(T=T_{(i) j}\right) .
$$

Proof By Fubini Theorem we can write

$$
\begin{aligned}
I_{(i) j} & =E\left\{\left[A_{(i) j}(T)-A_{(i) j}\left(Y_{(i) j}\right)\right]^{+}\right\} \\
& =E\left\{\int_{0}^{\infty} 1_{\left\{Y_{(i) j} \leq s<T\right\}} \mathrm{d}\left(-\ln \bar{F}_{(i) j}\left(s \wedge T_{(i) j}\right)\right)\right\} \\
& =E\left\{\int_{0}^{\infty}\left[\int_{0}^{t} 1_{\left\{Y_{(i) j} \leq s<T\right\}} \frac{\mathrm{d} F_{(i) j}(s)}{\bar{F}_{(i) j}(s)}\right] \mathrm{d} F_{(i) j}(t)\right\} \\
& \left.=E\left\{\int_{0}^{\infty}\left[\int_{0}^{\infty} \mathrm{d} F_{(i) j}(t)\right] 1_{\left\{Y_{(i) j} \leq s<T\right\}} \frac{\mathrm{d} F_{(i) j}(s)}{\bar{F}_{(i) j}(s)}\right]\right\} \\
& =E\left\{\int_{0}^{\infty} \bar{F}_{(i) j}(s) 1_{\left\{Y_{(i) j} \leq s<T\right\}} \frac{\mathrm{d} F_{(i) j}(s)}{\bar{F}_{(i) j}(s)}\right\} \\
& =E\left\{\int_{0}^{\infty} 1_{\left\{Y_{(i) j} \leq s<T\right\}} \mathrm{d} F_{(i) j}(s)\right\} \\
& =\int_{0}^{\infty} P\left(T=T_{(i) j} \mid T_{(i) j}=s\right) \mathrm{d} F_{(i) j}(s) \\
& =\int_{0}^{\infty} P\left(T=T_{(i) j}\right) \mathrm{d} F_{(i) j}(s) \\
& =P\left(T=T_{(i) j}\right) .
\end{aligned}
$$

As in the following proposition, we note that the identically and independent conditions are not essential.

Proposition 3.1.2 Let $T$ be the lifetime of a coherent system of components independent and identically distributed and let $T_{(i) j}$ be the $i$-th failure lifetime caused by the component $j$, with $\mathfrak{I}_{t}$-compensator process $\left(A_{(i) j}(t)\right)_{t \geq 0}$.

Then, the reliability importance of component $j$ to the system reliability at the $i$-th failures is

$$
I_{(i) j}=\int_{0}^{\infty} P\left(T=T_{(i) j} \mid T_{(i) j}=s\right) \mathrm{d} F_{(i) j}(s) .
$$

Proof As the process $1_{\left\{Y_{(i) j} \leq s<T\right\}}$ is $\mathfrak{I}_{t}$-predictable, we 
have

$$
\begin{aligned}
I_{(i) j} & =E\left\{\int_{0}^{\infty} 1_{\left\{Y_{(i) j} \leq s<T\right\}} \mathrm{d} A_{(i) j}(s)\right\} \\
& =E\left\{\int_{0}^{\infty} 1_{\left\{Y_{(i) j} \leq s<T\right\}} \mathrm{d} N_{(i) j}(s)\right\} \\
& =P\left(Y_{(i) j} \leq T_{(i) j}<T\right)=P\left(T=T_{(i) j}\right) \\
& =\int_{0}^{\infty} P\left(T=T_{(i) j} \mid T_{(i) j}=s\right) \mathrm{d} F_{(i) j}(s) .
\end{aligned}
$$

Example 3.1.1 Let $T$ be the system lifetime given by $T=T_{1} \wedge\left(T_{2} \vee T_{3}\right)$ of three Components independent and identically distributed lifetimes with absolutely continuous distribution function $F$. Follows that

$$
\begin{gathered}
Y_{(1) 1}=0 ; Y_{(1) 2}=Y_{(1) 3}=\infty ; \\
Y_{(2) 1}=T_{2} \wedge T_{3} ; Y_{(2) 2}=T_{3} ; Y_{(2) 3}=T_{2} .
\end{gathered}
$$

Therefore

$$
\begin{aligned}
I_{(1) 1} & =E\left\{\left[A_{(1) 1}(T)-A_{(1) 1}\left(Y_{(1) 1}\right)\right]^{+}\right\} \\
& =E\left\{\int_{0}^{\infty} 1_{\{0 \leq s<T\}} \mathrm{d} A_{(1) 1}(s)\right\}=E\left\{\int_{0}^{\infty} 1_{\{0 \leq s<T\}} \mathrm{d} N_{(1) 1}(s)\right\} \\
& =P\left(T_{(1) 1}<T\right)=P\left(T_{1}<T_{2} \wedge T_{3}\right)=\frac{1}{3} . \\
I_{(2) 1} & =E\left\{\int_{0}^{\infty} 1_{\left\{T_{2} \wedge T_{3} \leq s<T\right\}} \mathrm{d} A_{(2) 1}(s)\right\} \\
& =E\left\{\int_{0}^{\infty} 1_{\left\{T_{2} \wedge T_{3} \leq s<T\right\}} \mathrm{d} N_{(2) 1}(s)\right\} \\
& =P\left(T_{2} \wedge T_{3} \leq T_{(2) 1}<T\right) \\
& =P\left(T_{2} \wedge T_{3} \leq T_{1}<T_{2} \vee T_{3}\right)=\frac{1}{3} \\
I_{(2) 2} & =E\left\{\int_{0}^{\infty} 1_{\left\{T_{3} \leq s<T\right\}} \mathrm{d} A_{(2) 2}(s)\right\} \\
& =P\left(T_{3} \leq T_{(2) 2}<T\right)=P\left(T_{3} \leq T_{2}<T_{1}\right)=\frac{1}{6}
\end{aligned}
$$

Using symmetry we have $I_{(2) 3}=I_{(2) 2}=\frac{1}{6}$. We also have

$$
I_{(1)}=P\left(T=T_{(1)}\right)=I_{(1) 1}=\frac{1}{3}
$$

and

$$
\begin{aligned}
I_{(2)} & =P\left(T=T_{(2)}\right)=\frac{2}{3}=\frac{1}{3}+\frac{1}{6}+\frac{1}{6} \\
& =I_{(2) 1}+I_{(2) 2}+I_{(2) 3} .
\end{aligned}
$$

\subsection{Component Importance under Compensator Transforms}

To work in dependence conditions we consider a probability space $(\Omega, \mathfrak{I}, P)$ and a family of sub $\sigma$-algebras $\left(\mathfrak{J}_{t}\right)_{t \geq 0}$ of $\mathfrak{J}$ as in Section 2, which is increasing, right continuous and completed (shortly: satisfies the Dellacheries usual conditions).

Let $A_{k}(t)$ be the $\mathfrak{I}_{t}$-compensator of $N_{k}(t)=1_{\left\{T_{k} \leq t\right\}}$ where $T_{k}$ is a totally inaccessible $\mathfrak{J}_{t}$-stopping time representing the lifetime of the component $k$. Let $\left(\alpha_{k}(t)\right)_{t \geq 0}$ be a $\mathfrak{I}_{t}$-predictable non-negative process, interpreted as a hazard transformation process for $T_{k}$.

Usually $\alpha_{k}(t)$ can be associated with an improvement of $T_{k}$. Define

$$
B_{k}(t)=\int_{0}^{t} \alpha_{s} \mathrm{~d} A_{k}(s)
$$

such that $B_{k}(t)<\infty$. Under certain conditions, it is possible to find a new probability measure $P_{\alpha_{k}}$ such that $B_{k}(t)$ is the $\mathfrak{J}_{t}$-compensator of $N_{k}(t)$ under $P_{\alpha_{k}}$. Indeed, assume that the process

$$
L_{k}(t)=\alpha_{k}\left(T_{k}\right)^{N_{k}(t)} \exp \left(A_{k}(t)-B_{k}(t)\right)
$$

is uniformly integrable. Then, it follows from well known results on point process martingales, that $L_{k}(t)$ is an $\mathfrak{J}_{t}$-martingale with expectation 1 , and the desired measure $P_{\alpha_{k}}$ is given by

$$
\left(\frac{\mathrm{d} P_{\alpha_{k}}}{\mathrm{~d} P}\right)_{\mathfrak{I}_{t}}=L_{k}(t) .
$$

(See Jacod [13], Prop. 4.3 and Th 4.5; simple adaptation can be found in Arjas and Norros [14].)

At this point we can ask what are the effects of the independent (exchangeable) components distribution lifetimes transformation in the dependent ordered statistics and in the system signature representation itself. We remark that, in the following results, the proofs are heavily based in the fact that $\mathfrak{I}_{t}$-martingales summation is an $\mathfrak{I}_{t}$-martingale and the $\mathfrak{I}_{t}$-compensator is unique.

Theorem 3.2.1 Let $N_{k}(t)$ and $A_{k}(t)$ be as above. Under the compensator transform

$$
B_{k}(t)=\int_{0}^{t} \alpha_{s} \mathrm{~d} A_{k}(s)<\infty
$$

and under $P_{\alpha_{k}}$, the $\mathfrak{J}_{t}$-compensator of the $i$-th failure, $A_{i}(t)$, is transformed to

$$
B_{i}(t)=\sum_{j=1}^{n} B_{(i) j}(t)
$$

where 


$$
B_{(i) j}(t)=A_{(i) j}(t) \quad j \neq k
$$

and

$$
B_{(i) k}(t)=\int_{0}^{t} \alpha_{k}(s) \mathrm{d} A_{(i) k}(s) .
$$

Proof We observe that the $\mathfrak{I}_{t}$-compensator of the $i$-th failure is set as

$$
A_{i}(t)=\sum_{j=1}^{n} A_{(i) j}(t)=1_{\left\{T_{(i)} \leq t<T_{(i+1)\}}\right.} \sum_{j=1}^{n} A_{(i) j}(t) .
$$

Also, the component $\mathfrak{J}_{t}$-compensator can be set in the form:

$$
\begin{aligned}
A_{j}(t) & =\sum_{i=1}^{n} A_{(i) j}(t)=\sum_{i=1}^{n} 1_{\left\{T_{(i)} \leq t<T_{(i+1)}\right\}} A_{(i) j}(t) \\
& =\sum_{i=1}^{n} 1_{\left\{T_{(i)} \leq t<T_{(i+1)}\right\}} A_{j}(t) .
\end{aligned}
$$

In the case of the $\alpha_{k} \quad \mathfrak{I}_{t}$-compensator transform we have

$$
\begin{aligned}
B_{k}(t) & =\int_{0}^{t} \alpha_{k}(s) \mathrm{d} A_{k}(s)=\sum_{l=1}^{n} \int_{T_{(l-1)}}^{T_{(l)} \wedge t} \alpha_{k}(s) \mathrm{d} A_{k}(s) \\
& =\sum \int \alpha_{(l) k}(s) \mathrm{d} A_{(l) k}(s),
\end{aligned}
$$

with $T_{(0)}=0$, where $\alpha_{(l) k}(s)=1_{\left\{T_{(l-1)} \leq s<T_{(l)}\right\}} \alpha_{k}(s)$.

Therefore, the effect of the $\alpha_{k}$ compensator transform in the compensator of the ith failure is through the $i$-th term of the last summation.

$$
\begin{aligned}
B_{(i)}(t) & =\sum_{j=1}^{n} B_{(i) j}(t) \\
& =1_{\left\{T_{(i)} \leq t<T_{(i+1)}\right\}}\left[\sum_{j \neq k=1}^{n} B_{(i) j}(t)+\int_{T_{(i-1)}}^{T_{(i)} \wedge t} \alpha_{(i) k}(s) \mathrm{d} A_{(i) k}(s)\right]
\end{aligned}
$$

Assuming that $L_{k}(t)$ is uniformly integrable,

$E\left[L_{k}\left(T_{k}\right)\right]=1$ and, under $P_{\alpha_{k}}$ with $\left(\frac{\mathrm{d} P_{\alpha_{k}}}{\mathrm{~d} P}\right)_{\mathfrak{I}_{t}}=L_{k}(t)$ is the $\mathfrak{J}_{t}$-compensator of $N_{(i)}(t)=1_{\left\{T_{(i)} \leq t\right\}}$ and the effect of the $\alpha_{k}$ compensator transform, in the compensator of the $i$-th failure is

$$
B_{(i) k}(t)=\int_{0}^{t} \alpha_{(i) k}(s) \mathrm{d} A_{(i) k}(s) .
$$

Based in the Bergman [1] notion of reliability importance and in Theorem 3.1.1, we can define.

Definition 3.2.1 Let $T$ and $T_{k}$ be the system and the $k$-th component lifetimes respectively and let $P_{\alpha_{k}}$ be defined as above (assuming that $L_{k}(t)$ is uniformly integrable). Suppose further that $T_{k}$ is finite and $T$ is integrable. Then the $\alpha_{k}$ importance of $T_{k}$ for $T$ in $\left\{T=T_{(i)}\right\}$ is

$$
\begin{aligned}
I_{(i) \alpha_{k}} & =E_{P_{\alpha_{k}}}[T]-E_{P}[T]=\operatorname{COV}\left(T, L_{k}(\infty)\right) \\
& =\operatorname{COV}\left(T, L_{k}\left(T_{(i) k}\right)\right)
\end{aligned}
$$

where $T_{(i) j}$ is the lifetime of the $i$-th failure caused by the $j$-th component failure.

Examples 3.2.1 1) Consider the compensator change which arises from exactly one minimal repair of component $k$ (Natvig [8]). Intuitively this means that when $T_{k}$ occurs, the system is returned to the state in which it was immediately before $T_{k}$ occurred. The second occurrence of $T_{k}$ is considered as final, and we take it as the improved value of $T_{k}$. This improvement operation corresponds to the compensator transformation (Norros [15])

$$
B_{k}(t)=\int_{0}^{t} \frac{A_{k}(s)}{1+A_{k}(s)} \mathrm{d} A_{k}(s)=A_{k}(t)-\ln \left(1+A_{k}(t)\right) .
$$

In this case

$$
\alpha_{k}(s)=\frac{A_{k}(s)}{1+A_{k}(s)}
$$

and therefore

$$
L_{k}\left(T_{k}\right)=\alpha_{k}\left(T_{k}\right)^{N_{k}(t)} \exp \left(A_{k}(t)-B_{k}(t)\right)=A_{k}\left(T_{k}\right)
$$

and, therefore,

$$
I_{(i) \alpha_{k}}=-\operatorname{COV}\left(T, A_{(i) k}\left(T_{(i) k}\right)\right) .
$$

2) Bueno and Carmo [16] propose a parallel transform for the case of dependent components, through compensator transform as

$$
\begin{aligned}
B_{k}(t) & =\int_{0}^{t}\left(\frac{2-2 \exp \left[-A_{k}(s)\right]}{2-\exp \left[-A_{k}(s)\right]}\right) \mathrm{d} A_{k}(s) \\
& =A_{k}(t)-\ln \left(2-\exp \left[-A_{k}(t)\right]\right) .
\end{aligned}
$$

That is, we consider the transformations where

$$
\begin{gathered}
\alpha_{k}(s)=\frac{2-2 \exp \left[-A_{k}(s)\right]}{2-\exp \left[-A_{k}(s)\right]}, \\
L_{k}\left(T_{k}\right)=2-2 \exp \left[-A_{k}\left(T_{k}\right)\right]
\end{gathered}
$$

and

$$
I_{(i) \alpha_{k}}=-\operatorname{COV}\left(T, 2-2 \exp \left[-A_{(i) k}\left(T_{(i) k}\right)\right]\right) .
$$

The case of deterministic compensators

Under the assumptions that $T_{i}, 1 \leq i \leq n$, are totally 
inaccessible $\mathfrak{I}_{t}$-stopping time, the $\mathfrak{I}_{t}$-compensators are continuous and from Arjas and Yashin [12] we conclude that

$$
A_{(i) k}(t)=-\ln \left(\bar{F}_{(i) k}\left(t \wedge T_{(i) k}\right)\right) .
$$

If $A_{i(j)}(t)$ is a determinist increasing function of $t$ (except that it is stopped when $T_{(i) k}$ occurs) we could say that $T_{(i) k}$ is dynamically independent of everything else in the history $\mathfrak{I}_{t}$. This is a generalization of the case of independent components: if the component lifetime $T_{(i) k}$ has a continuous compensator which is deterministic, then the lifetimes of the others components have no causal effect in $T_{(i) k}$. However, other components may well dependent casually on $T_{(i) k}$, so that the components need not be statistically independent.

Lemma 3.2.1 Assume that $L_{k}$ is a $\sigma\left(T_{k}\right)$-measurable density $\left(E\left[L_{k}\right]=1\right)$. Denote

$$
F_{(i) k}(t)=P\left(T_{(i) k} \leq t\right)
$$

and

$$
F_{(i) k}^{*}(t)=P_{L k}\left(T_{(i) k} \leq t\right) .
$$

Suppose that $E[T]<\infty$ and $T_{(i) k}$ Is finite. Then

$$
\begin{aligned}
I_{(i) \alpha_{k}} & =E\left[L_{k} T\right]-E[T] \\
& =\int_{0}^{\infty}\left(\bar{F}_{(i) k}^{*}(t)-\bar{F}_{(i) k}(t)\right) I_{t}^{B}\left(T_{(i) k}, T\right) \mathrm{d} t .
\end{aligned}
$$

Proof

$$
\begin{aligned}
E[ & \left.L_{k} T\right]=E\left\{L_{k} E\left[T \mid T_{(i) k}\right]\right\}=E\left\{L_{k} \int_{0}^{\infty} P\left(T>s \mid T_{(i) k}\right) \mathrm{d} t\right\} \\
= & \int_{0}^{\infty}\left[F_{(i) k}^{*}(t) P\left(T>t \mid T_{(i) k} \leq t\right)\right. \\
& \left.+\bar{F}_{(i) k}^{*}(t) P\left(T>t \mid T_{(i) k}>t\right)\right] \mathrm{d} t \\
= & \int_{0}^{\infty} F_{(i) k}^{*}(t)\left[P\left(T>t \mid T_{(i) k}>t\right)-P\left(T>t \mid T_{(i) k} \leq t\right)\right] \mathrm{d} t \\
& -\int_{0}^{\infty} P\left(T>t T_{(i) k} \leq t \mid\right) \mathrm{d} t .
\end{aligned}
$$

The last integral is finite by the assumptions. The results follow by applying the above formula also for $L_{k}=1$ and subtracting.

Examples 3.2.2 1) As in example 3.31 ) consider the compensator change which arises from exactly one minimal repair. Follows that, the compensator transform of $A_{(i) k}(t)$ is

$$
B_{k}(t)=A_{k}(t)-\ln \left(1+A_{k}(t)\right) .
$$

As

$$
A_{(i) k}(t)=-\ln \left(\bar{F}_{(i) k}\left(t \wedge T_{(i) k}\right)\right),
$$

in the set $\left\{T_{(i) k}>t\right\}$, we have

$$
\begin{aligned}
B_{(i) k}(t) & =-\left[\ln \bar{F}_{(i) k}(t)+\left(1-\ln \bar{F}_{(i) k}(t)\right)\right] \\
& =-\ln \left[\bar{F}_{(i) k}(t)\left(1-\ln \bar{F}_{(i) k}(t)\right)\right]
\end{aligned}
$$

and

$$
\bar{F}_{(i) k}^{*}(t)=\exp \left[-A_{(i) k}(t)\right]=\bar{F}_{(i) k}(t)-\bar{F}_{(i) k}(t) \ln \bar{F}_{(i) k}(t) .
$$

Therefore

$$
I_{(i) \alpha_{k}}=\int_{0}^{\infty} \bar{F}_{(i) k}(t) \ln \bar{F}_{(i) k}(t) I_{t}^{B}\left(T_{(i) k}, T\right) \mathrm{d} t .
$$

2) In the case of Example 3.3 2) we have a parallel transform which transform $A_{(i) k}(t)$ to

$$
B_{k}(t)=A_{k}(t)-\ln \left(2-\exp \left[-A_{k}(t)\right]\right)
$$

Now, in the set $\left\{T_{(i) k}>t\right\}$, we have

$$
\begin{aligned}
B_{(i) k}(t) & =-\left[\ln \bar{F}_{(i) k}(t)+\ln \left(2-\bar{F}_{(i) k}(t)\right)\right] \\
& =-\ln \left[\bar{F}_{(i) k}(t)+\bar{F}_{(i) k}(t) F_{(i) k}(t)\right]
\end{aligned}
$$

Therefore

$$
F_{(i) k}^{*}(t)=\left[\bar{F}_{(i) k}(t)+\bar{F}_{(i) k}(t) F_{(i) k}(t)\right]
$$

and

$$
I_{(i) \alpha_{k}}=\int_{0}^{\infty} \bar{F}_{(i) k}(t) F_{(i) k}(t) I_{t}^{B}\left(T_{(i) k}, T\right) \mathrm{d} t .
$$

\section{Acknowledgements}

This work was partially supported by FAPESP, Proc. No. 2010/52227-0.

\section{References}

[1] B. Bergman, "On Some New Reliability Importance Measures,” In: W. J. Quirk, Ed., Proceedings of the 4th IFAC Workshop on Safety of Computer Control Systems (IFAC SAFECOMP), Como, 1-3 October 1985, pp. 6164.

[2] F. Samaniego, "System Signatures and Their Applications in Engineering Reliability,” International Series in Operation Research and Management Science, Vol. 110, Springer, New York, 2007.

[3] F. Samaniego, "On Closure of the IFR Class under Formation of Coherent Systems," IEEE Transactions on Reliability, Vol. R-34, No. 1, 1985, pp. 69-72. doi:10.1109/TR.1985.5221935

[4] S. Kochar, H. Mukherjee and F. Samaniego, “The Signa- 
ture of a Coherent System and Its Application to Comparisons among Systems,” Naval Research Logistic, Vol. 46, No. 5, 1999, pp. 507-523. doi:10.1002/(SICI)1520-6750(199908)46:5<507::AID-N AV4>3.0.CO;2-D

[5] M. Shaked and A. Suarez-Llorens, "On the Comparison of Reliability Experiments Based on the Convolution Order," Journal of American Statistical Association, Vol. 98, No. 4, 2003, pp. 693-702. doi:10.1198/016214503000000602

[6] Z. W. Birnbaum, "On the Importance of Different Components in a Multicomponent System,” In: P. R. Krishnaiah, Ed., Multivariate Analysis, Academic Press, New York, 1969, pp. 591-592.

[7] R. Barlow and F. Proschan, "Importance of Systems Components and Fault Trees Events," Stochastic Processes and Their Applications, Vol. 3, No. 2, 1975, pp. 153-173.

[8] B. Natvig, "New Light on Measure of Importance of Systems Components," Scandinavian Journal of Statistics, Vol. 12, No. 1, 1985, pp. 43-54.

[9] P. J. Boland and E. El-Neweihi, "Measures of Component Importance in Reliability Theory,” Technical Report, Florida State University, Tallahassee, 1990.

[10] V. C. Bueno, "Dynamics Signature of a Coherent System,” Technical report, São Paulo University, São Paulo,
2010.

[11] T. Aven and U. Jensen, "Stochastic Models in Reliability,” Springer Verlag, New York, 1999. doi:10.1198/016214503000000602

[12] E. Arjas and A. Yashin, "A Note on Random Intensities and Conditional Survival Functions,” Journal of Applied Probability, Vol. 25, No. 3, 1988, pp. 630-635. doi:10.1198/016214503000000602

[13] J. Jacod, "Multivariate Point Processes: Predictable Projection. Radon-Nikodyn Derivatives, Representation of Martingales," Zeitschrift fur Wahrscheinlichkeitstheorie und Verwandte Gebiete, Vol. 31, 1975, pp. 235-253. doi:10.1007/BF00536010

[14] E. Arjas and I. Norros, "Change of Life Distribution via a Hazard Transformation: An Inequality with Application to Minimal Repair,” Mathematics of Operations Research, Vol. 14, No. 2, 1989, pp. 355-361.

[15] I. Norros, "Notes on Natvig's Measure of Importance of Systems Components," Scandinavian Journal of Statistics, Vol. 12, 1986, pp. 43-54.

[16] V. C. Bueno and I. M. Carmo, "Active Redundancy Allocation for a k-out-of-n:F System of Dependent Components," European Journal of Operational Research, Vol. 176, No. 2, 2007, pp. 1041-1051. doi:10.1016/j.ejor.2005.09.012 